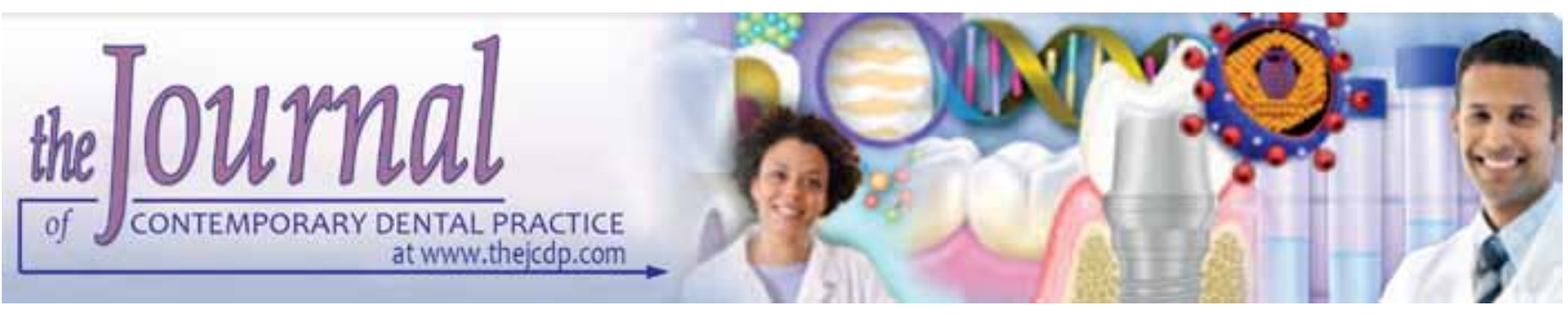

\title{
Quantitative Evaluation of Apical Extrusion of Debris and Irrigants using Four Rotary Instrumentation Systems: An in vitro Study
}

\author{
S Aspalli Nagaveni, K Reddy Balakoti, Karan Smita, P Ratnakar, SV Satish, T Aravind
}

\begin{abstract}
Clinical significance: The apical extrusion of infected debris may have the potential to disrupt the balance between microbial aggression and host defense, resulting in incidents of acute inflammation. During preparation, irrigants and debris, such as bacteria, dentin filings and necrotic tissue may be extruded into the periradicular region leading to periapical inflammation and postoperative flare ups. Using an instrumentation technique that minimizes apical extrusion would be beneficial to both the practitioner and patient.

The purpose of the study was to evaluate the weight of debris and volume of irrigant extruded apically from extracted teeth in vitro after endodontic instrumentation using four different rotary root canal instrumentation systems.
\end{abstract}

Materials and methods: Four groups of each 20 extracted mandibular premolars were instrumented using one of the four systems: ProTaper Universal (Dentsply Maillefer, Ballaigues, Switzerland)), Hero-shaper (MicroMega, Besancon, France), RaCe (FKG Dentaire, La-Chaux-de-Fonds, Switzerland) and K3 (SybronEndo, West Collins, CA). Debris and irrigant extruded from the apical foramen during instrumentation were collected in preweighed test tubes. Volume of irrigant extruded was noted. The containers were stored in incubator at $70^{\circ}$ for two days to evaporate the moisture. Weight of dry debris was noted.

Statistical analysis: Data was analyzed using Kruskall-Wallis and Mann-Whitney $U$ test at a significance of 0.001 .

Results: The results indicated that all of the instrumentation systems tested caused measurable apical extrusion of debris and irrigants. Higher extrusion was observed with Protaper system which was statistically significant with Hero-Shaper, $\mathrm{RaCe}$ and $\mathrm{K} 3$ systems. There were no statistical differences between Hero-shaper, K3 and RaCe systems $(p<0.05)$.

Conclusion: All instrumentation techniques apically extruded debris and irrigant. However, Hero-shaper, K3 and RaCe systems produced less extruded debris and irrigant than the Protaper system.

Keywords: Canal preparation, Rotary instruments, Apical extrusion, Debris, Irrigant.
How to cite this article: Nagaveni SA, Balakoti KR, Smita K, Ratnakar P, Satish SV, Aravind T. Quantitative Evaluation of Apical Extrusion of Debris and Irrigants using Four Rotary Instrumentation Systems: A in vitro study. J Contemp Dent Pract 2013;14(6):1065-1069.

Source of support: Nil

Conflict of interest: None

\section{INTRODUCTION}

Successful cleaning and shaping of root canal is dependent on adherence to specific biologic and mechanical objectives. The ultimate object of canal preparation is the elimination of irritant factors and maintenance of healthy periapical tissues. During preparation, irrigant and debris, such as bacteria, dentin filings and necrotic tissue, may be extruded into the periradicular region leading to periapical inflammation and postoperative flare ups. ${ }^{1}$

The apical extrusion of infected debris may have the potential to disrupt the balance between microbial aggression and host defence, resulting in incidents of acute inflammation and flare ups. ${ }^{2}$

Both contaminated and uncontaminated dentin and pulp tissue can trigger an inflammatory reaction when forced periapically during instrumentation. ${ }^{3}$ The immunologic aspects of postoperative flare ups were assessed by a number of researchers who concluded that antigens originating in the root canal result in the formation of antigen antibody complex which, when forced beyond the apical foramen, can lead to a severe inflammatory response. ${ }^{4-6}$

Therefore, it is logical to assume that minimizing the amount of apically extruded material should reduce postoperative reactions. For cleaning and shaping of root canals, various hand and rotary endodontic instruments are required. Vande Vise and Brilliant first quantified the 
amount of debris apically extruded during instrumentation. They found that all the canals in the presence of irrigating solution showed collectible aspirants of extruded material, whereas instrumentation of canals without an irrigant did not produce collectible extruded material. However, other inherent problems existed with dry instrumentation and irrigation was deemed essential. ${ }^{7}$

In 1982, Martin and Cunningham showed that endosonic instrumentation produced less apically extruded material than did hand filing. ${ }^{8}$ In 1988 , Fairbourn and Walter found that sonic, ultrasonic and cervical flaring instrumentation techniques produced less debris than conventional filing technique. ${ }^{9}$ In 1990, Douglas revealed that balanced force technique extruded significantly less debris than either endosonic or step back techniques. No significant difference was demonstrated between endosonic and step back filing techniques. $^{10}$

Al Omar and Dummer, in 1995, revealed that techniques involving a filing (linear motion) caused significantly more apical dentin debris than those involving some sort of rotational action. ${ }^{6}$ Reddy and Hicks were the first to compare apical debris extrusion between manual instrumentation and engine driven techniques. When comparing the mean weights of apically extruded debris, they noted that the step back technique produced significantly more debris than the engine driven techniques and balanced force technique. Rotary instruments have a tendency to pull dentinal debris into the flutes of the file and direct it toward the coronal aspect of the canal. ${ }^{11}$

During the last decade, root canal preparations with rotary NiTi instruments have become popular. Because canal instrumentation with rotary NiTi systems remain more centered in the root canal, this results in less transportation of materials than hand filing with stainless steel files. Also, this type of instrumentation is more comfortable for patients and operators. Nowadays, advanced instrument designs including specific tip geometries, alternating cutting edges, varying tapers, changing pitch lengths and surface treatments have been developed to improve efficacy and safety. ${ }^{12,13}$

The purpose of this study was to quantitatively evaluate and compare the apical extrusion of debris and irrigant using four rotary instrument systems, ProTaper Universal, Hero Shaper files, RaCe and K3.

\section{MATERIALS AND METHODS}

Eighty freshly extracted human single rooted mandibular premolars with mature apices and curvature of 0 to $10^{\circ}$ were selected. All of these teeth had a single root canal and one apical foramen evident in radiographs. Teeth with calcification and open apices were excluded. The curvatures of experimental teeth were measured using Schneider's method. Teeth were cleansed and stored in a screw top bottle containing sterile distilled water to which thymol was added to act as disinfectant. Coronal access was prepared conventionally with a high speed round bur and the pulp remnants were removed with a barbed broach. Eighty teeth in which $15 \mathrm{~K}$ file could bind at the working length were selected. They had straight root canals of similar size to reduce effects of canal size and curvature on the extrusion of debris and irrigant.

Tooth length was standardized to $15 \mathrm{~mm}$. They were randomly assigned into four groups with 20 specimens in each. The teeth were forced through a precut hole in the rubber stopper. Before canal instrumentation, test tubes were preweighed to $10^{-5} \mathrm{gm}$ precision. During the measurement, three consecutive readings were taken and the average value was recorded. The rubber stopper with the tooth was then fitted into the mouth of the test tube. A 19 gauge needle was inserted next to the inserted root to equalize the external and internal pressures. The experimental teeth were numbered randomly. The numbers were marked both on the test tubes and the rubber stopper. The tube was hand held vertically during instrumentation. The system was sealed with rubber dam to avoid leakage of the irrigant into the tube.

\section{IRRIGATION PROCEDURE}

Distilled water was used as irrigant. Around $0.5 \mathrm{ml}$ of distilled water was deposited into the canal before initiating any instrumentation, and $0.5 \mathrm{ml}$ of distilled water was used after each instrumentation using 28 gauge needle. The needle was placed as far into the canal as possible without binding. The canals of each group were prepared by one of the following techniques using the specific instruments.

- Group I: Instrumented with ProTaper rotary system.

- Group II: Instrumented with Hero-Shaper file system.

- Group III: Instrumented with RaCe system.

- Group IV: Instrumented with K3 system.

Preparation in all groups was done using X-Smart (Dentsply), and the canals were enlarged apically till 30 size 0.04 taper.

Immediately upon completion of the instrumentation procedure, the volume of irrigant extruded was noted. Then tooth with rubber stopper was removed from the test tube and debris adhering to the root surface were collected by washing the apex with $1 \mathrm{ml}$ of distilled water into the test tube. The test tubes were stored in incubator at $70^{\circ} \mathrm{C}$ for 2 days to evaporate the moisture. Weighing was carried out on an electronic microbalance and repeated for three times, and the mean value was recorded. Mean first weights were compared with mean second weights and the difference was recorded as the weight of extruded debris. 


\section{STATISTICAL ANALYSIS}

Descriptive data included mean and standard deviation. Statistical comparisons were performed using KruskalWallis test for multiple groups and Mann-Whitney U test for pairwise comparison.

\section{RESULTS}

The results indicated that all of the instruments tested caused measurable apical extrusion of debris and irrigants (Graphs 1 and 2) (Table 1). Higher extrusion was observed with ProTaper system which was statistically significant with Hero-shaper, RaCe and K3 systems. There was no statistical difference between Hero- shaper, RaCe and K3 systems $(\mathrm{p}<0.05)$ (see Table 1$)$.

\section{DISCUSSION}

Torabinejad et al stated that physical or chemical injury of periradicular tissues during cleaning and shaping of the root canal system can cause degranulation of mast cells in periapical tissues. Mast cells discharging vasoactive amines into the periapical tissues initiate an inflammatory response or aggravate an existing inflammatory process. ${ }^{3}$

Many researchers have looked at various aspects of apically extruded debris and suggested that no technique is totally effective in preventing debris extrusion. On other hand, it can be argued that greater the debris more severe could be the reaction. ${ }^{3,14}$

There are few studies which compared 0.04 and 0.06 taper nickel titanium instruments with conventional hand instrumentation technique. ${ }^{11,15,16,17}$ There have been few

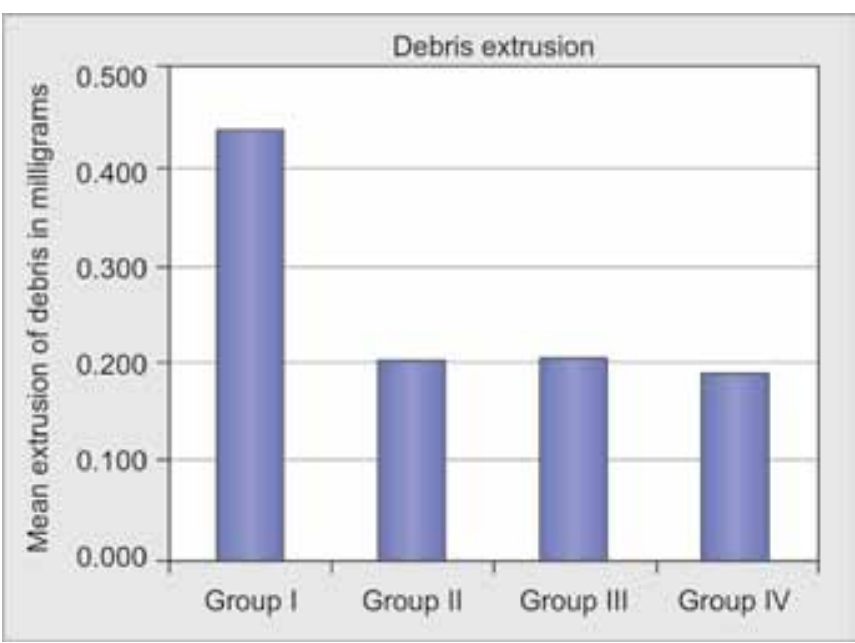

Graph 1: Comparison of apical extrusion of debris in four groups published studies to date on NiTi instruments ProTaper, Hero-shaper, K3 and RaCe systems concerning apical debris and irrigant extrusion in extracted human teeth. Hence, these four experimental groups were selected in this study.

Great care was taken to ensure that the experimental groups balanced with respect to canal shape. The variations recorded in terms of weight of debris were attributed entirely to difference in preparation techniques. Pulp tissue remnants were removed before preparation and had no influence on results. Common to all techniques were the amount and type of irrigant and the operator. Each sample was prepared using new set of instruments to ensure that wear of instrument had no influence over debris extrusion.

Distilled water was chosen as the irrigant to reduce the chance that particulate matter dwelling in other irrigants might possibly skew the final values. Density of water is one. So, volume of water is equal to the weight (mass) of water. So, the volume of irrigant extruded can be directly known by weighing water which simplifies the procedure. The passive injection of irrigant was done because preliminary data indicated that passive injection minimizes oozing debris and irrigant out of the apical foramen with anything other than the filing system being tested.

Microelectronic balance was selected because the samples were so small that a sensitive microbalance was required to weigh them. The weight of sample would increase due to hydration from moisture in the air as the sample was being weighed. Hence, the microbalance used in our study had a protective glass cover to prevent dehydration of the sample.

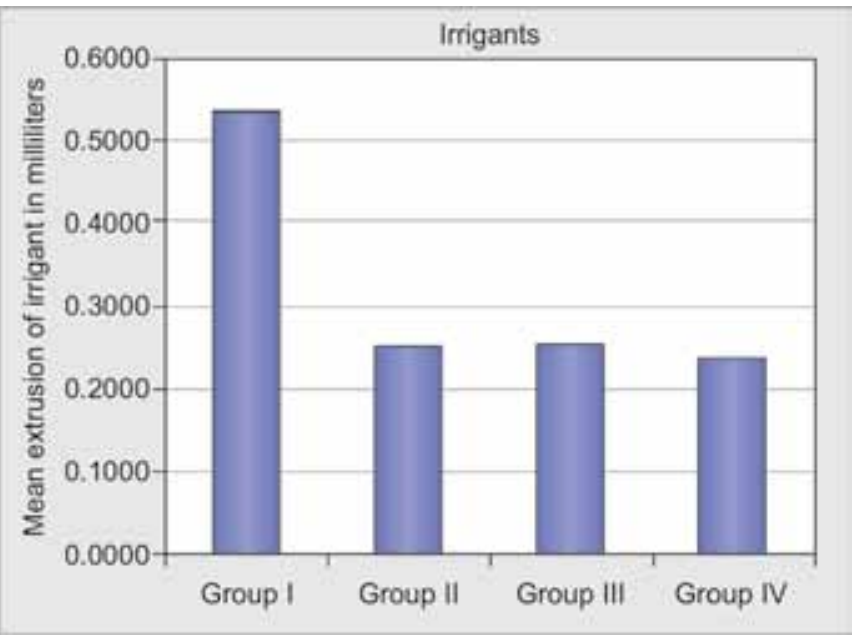

Graph 2: Comparison of apical extrusion of irrigant in four groups

\begin{tabular}{lllllll}
\multicolumn{7}{c}{ Table 1: Comparison of mean apical extrusion of debris and irrigants in four groups } \\
\hline Parameter & Group I & Group II & Group III & Group IV & p-value & Significance \\
\hline Mean debris extrusion & 0.437 & 0.198 & 0.198 & 0.184 & $<0.001$ & HS \\
Mean irrigant & 0.5349 & 0.2502 & 0.2497 & 0.2334 & $<0.001$ & HS
\end{tabular}

HS: Highly significant 
It was showed that if infected dentin chips are accumulated between the filling material and the periapical tissues, the healing process may be impaired. ${ }^{18}$ It was also noted that if dentin chips formed the plug, more apical inflammation and less healing occurred compared to teeth without plugs.

The hypothesis that engine driven rotary NiTi instruments will produce less debris than hand filing was strongly supported. ${ }^{9-12}$ This could be accounted to the file design of NiTi instruments which enlarge the cervical region of the canal prior to preparing the apical part. The advantages of early cervical flaring are as follows:

- Deeper penetration of irrigation solution and easy removal of debris from apical area

- Reduced possibility of ledging

- Reduced possibility of debris packing

- The bulk of canal contents are removed before apical instrumentation, greatly reducing the number of contaminants that can be extruded from canal.

The unique design features of NiTi files encourage coronal rather than apical displacement of debris during preparation process. The flat outer edges keep the file centered in the canal and interflute distance tends to accumulate dentinal debris and directs it coronally.

The reasons that can be attributed to less extrusion of debris in $\mathrm{K} 3$ is presence of increasing helical flute angle which gives the instrument the benefit of unparalleled debris removal. ProTaper system caused a significantly large amount of extruded debris because it is a faster, aggressive system with its characteristic design features, which removes a substantial amount of dentin in a shorter period of time, is unable to coronally displace the debris with the same efficiency as it cuts and thus poses a risk of increased apical extrusion of debris and irrigant.

$\mathrm{RaCe}$ instruments have nonconvex triangular crosssectional design and smaller core diameter, which allows more space to carry debris out toward the orifice, thus avoiding its compaction in the root canal. S1, S2, F1 and F2 ProTaper universal instruments have convex triangular cross-section, their debris space is smaller than that of RaCe. $\mathrm{RaCe}$ instruments also have short twisted cutting edges alternating with straight edges, may give rise to favorable debris transporting spaces. Less extrusion in Hero-shapers is attributed to less number of files used. Only two rotary files complete the canal preparation whereas ProTaper has many instruments.

The results presented here are consistent with other investigations. Mohammad HZ, Maryam B compared apically extruded debris from three rotary instrumentation techniques (Profile, RaCe, FlexMaster) and concluded that $\mathrm{RaCe}$ extruded the least debris followed by Profile and more debris extrusion was noted with Flex Master which was statistically significant with RaCe. ${ }^{19}$

Jale T, Figen K et al compared apically extruded debris between ProTaper, Profile and Hero-shaper file system. They conclude that profile extruded the least debris followed by Hero-shaper and more debris was noted with ProTaper which was statistically significant with profile system. ${ }^{20}$ Alper K, Kerem $\mathrm{E}$ et al compared extrusion of intracanal debris and irrigant following use of various systems and concluded that least values noted with $\mathrm{K} 3{ }^{21}$

\section{CONCLUSION}

The results of this study should be extrapolotated to clinical situations with caution. No attempt has been made to stimulate the presence of vital pulp or periapical tissues which may resist apical extrusion of debris in vivo.

\section{REFERENCES}

1. Seltzer S, Naidorf IJ. Flare-ups in endodontics: I. etiological factors. J Endod 1985;11:472-478.

2. Siqueira JF Jr. Microbial causes of endodontic flare-ups. Int Endod J 2003;36:453-463.

3. Ruiz-Hubard EE, Gutman Jl, Wagner MJ. A quantitative assessment of canal debris forced periapically during root canal instrumentation using two different techniques. J Endod 1987; 13:554-558.

4. Naidorf IJ. Endodontic flare ups: bacteriological and immunological mechanisms. J Endod 1985;11:462-464.

5. Torabinejad M, Eby WC, Naidorf IJ. Inflammatory and immunological aspects of the pathogenesis of human periapical lesions. J Endod 1985:479-488.

6. Al-Omari MAO, Dummer PMH. Canal blockage and debris extrusion with eight preparation techniques. J Endod 1995;21:154-158.

7. Vande Visse JE, Brilliant D. Effect of irrigation on the production of extruded material at the root apex during instrumentation. J Endod 1975;1:243-241.

8. Martin H, Cunningham WT. The effect of endosonic and hand manipulation on the amount of root canal material extruded. Oral Surg 1982;53:611-613.

9. Fairbourn DR, Walter GM. The effect of four preparation techniques on the amount of apically extruded debris. J Endod 1987;13:102-108.

10. Douglas JM. Comparison of balanced force, endosonic and step back filing instrumentation techniques: Quantification of extruded apical debris. J Endod 1990;16:24-28.

11. Apical extrusion of debris using two hand and two rotary instrumentation techniques. J Endod 1998;24:180-183.

12. Tasdemir T, Aydemir H, Inan U, Unal O. Canal preparation with Hero 642 rotary NiTi instruments compared with stainless steel hand $\mathrm{K}$-file assessed using computed radiography. Int Endod J 2005;38:402-408.

13. Lopez FU, Fachin EV, Camargo Fontanella VR, Barletta FB, So MV, Grecca FS. Apical transportation: a comparative evaluation of three root canal instrumentation techniques with three different apical diameters. J Endod 2008;34:1545-1548.

14. Sveg TA, Harrison JW. The effect of effervescence on debridement of the apical regions of root canals in single rooted teeth. J Endod 1981;7:335-341. 
15. Ferraz CR, Gomes NV. Apical extrusion of debris and irrigants using two hand and three engine driven instrumentation techniques. Int Endod J 2001;34:354-348.

16. Beeson T, Hartwel GR. Comparison of debris extruded apically in straight canals. Conventional filing verses Profile 04 taper series 29. J Endod 1998;24:18-24.

17. Hinrichs RE, Walker WA. Comparison of amount of apically extruded debris using hand piece driven nickel titanium instrument system. J Endod 1998;21:102-118.

18. Holland R, Souza VA. Tissue reactions following apical plugging of the root canal with infected dentin chips. Oral Surgery 1980;49:366-371.

19. Mohammad HZ, Maryam B. An in vitro comparative study of apically extruded debris resulting from conventional and three rotary (Profile, RaCe, FlexMaster) instrumentation techniques.

20. Jale T, Figen K. Quantitative evaluation of the amount of apically extruded debris using 3 different rotary instrumentation systems. Oral Surg 2006;101:250-257.

21. Alper K, Kerem E. Apical extrusion of intracanal debris and irrigant following use of various instrumentation techniques. Oral Surg 2008;105:257-262.

\section{ABOUT THE AUTHORS}

\section{S Aspalli Nagaveni (Corresponding Author)}

Assistant Professor, Department of Conservative Dentistry and Endodontics, AME's Dental College, Hospital and Research
Centre, Bijangere Road, Raichur-584103, Karnataka, India, e-mail: drnagaveniaspalli@gmail.com

\section{K Reddy Balakoti}

Professor and Head, Department of Conservative Dentistry and Endodontics, St Joseph Dental College and Hospital, Eluru, Andhra Pradesh, India

\section{Karan Smita}

Reader, Department of Periodontics, Adesh Institute of Dental Sciences Bathinda, Punjab, India

\section{P Ratnakar}

Reader, Department of Conservative Dentistry and Endodontics S. Nijalingappa Institute of Dental Sciences and Research Centre Gulbarga, Karnataka, India

\section{SV Satish}

Professor, Department of Conservative Dentistry and Endodontics Navodaya Dental College, Raichur, Karnataka, India

\section{T Aravind}

Professor, Department of Periodontics, Rama Dental College, Kanpur Uttar Pradesh, India 